\title{
Inter- and Intramolecular Interactions of Polymers as Studied by Fluorescence Spectroscopy. VIII. Absorption and Fluorescence Spectra of Polymer- Bound Anthryl Groups Both in Solution and Solid
}

\author{
Shigeo TAZuKe and Naoya HAYASHI \\ Research Laboratory of Resources Utilization, Tokyo Institute of Technology, \\ 4259 Nagatsuta, Midori-ku, Yokohama 227, Japan.
}

(Received November 28, 1977)

\begin{abstract}
The following five polymers bearing anthryl groups were prepared starting from 2-(9-anthrylmethyl)-1, 3-propanediol or bis (2-hydroxyethyl) (9-anthrylmethyl) malonate; poly[oxy(2-(9-anthrylmethyl)trimethylene)oxysuccinyl](PE(A-2)), poly[oxy(2-(9-anthrylmethyl) trimethylene)oxysebacyl](PE(A-8)), poly[oxy(2-(9-anthrylmethyl)trimethylene)oxyterephthaloyl](PE(A-Ph)), poly[oxy(9-anthrylmethyl)malonyloxyethyleneoxycarbonyliminohexamethyleneiminocarbonyloxyethylene](PU(A-6)), and poly[oxy(9-anthrylmethyl)malonyloxyethyleneoxycarbonylimino( $m$-xylylene)iminocarbonyloxyethylene](PU(A-Xy)).

Absorption spectra of these polymers in dilute solutions are nearly identical to that of the monomer model compounds, 1, 3-bisacetoxy-2-(9-anthrylmethyl)propane except for a slight broadening of the spectra of polymers. In the solid state, the absorption spectra indicate the presence of dimers and/or aggregated states of anthryl groups in the ground state. Fluorescence spectra of the polymers in solution consist of monomeric and intramolecular excimer emission. The contribution of excimer emission decreases by increasing the spacing between anthryl groups. However, a broad structureless emission peaking at about $460 \mathrm{~nm}$ alone was observed in the solid film except for PE(A-Ph) whose emission peak appeared at about $490 \mathrm{~nm}$. The excitation spectra in the solid state were broader than those in solution, indicating the importance of ground state interactions. The tendency of ground state interaction is much stronger for $\mathrm{PE}(\mathrm{A}-\mathrm{Ph})$ since the excitation spectrum of the polymer is almost structureless and does not agree with the absorption spectrum of anthryl group at all.

KEY WORDS: Absorption Spectroscopy / Fluorescence Spectroscopy / Interpolymer Interaction / Intrapolymer Interaction / Anthracene / Excimer / Solid State / Polyester / Polyurethane / Activated Ester /
\end{abstract}

In the series of articles on fluorescence spectroscopy of polymer-bound chromophores, ${ }^{1-6}$ we have shown that (i) excited state interactions are good indices representing solution properties of polymers, (ii) interpolymer interaction is a common feature in dilute solution particularly for exciplex forming polymers, (iii) the polymer main chain plays an important role in protecting the exciplex from solvation and dissociation, (iv) molecular weight affects both inter- and intramolecular interactions, and (v) the solvent effects provide qualitative information on the conformation of polymers in solution. In these studies, efforts were directed to the elucidation of polymer effects by comparison of monomer and dimer model compounds in dilute solutions.
In this article, we intend to compare solution and solid-state spectroscopies. When these photoresponsive polymers are considered as functional materials, solid-state properties are more important relevant to photopolymer applications. We have chosen the anthryl group as chromophore because of its unique spectroscopic properties as well as of its photoreactivity. The polyesters with pendant anthryl groups were studied for their photoreactivity and spectroscopic properties in solution. ${ }^{7}$ The results indicated that sufficient conditions for efficient excimer formation in polymeric systems are the high local concentration of chromophores and the mobility of chromophores which makes possible the build up of a favorable conformation for the excimer within the 


\section{S. TAZUKE and N. HAYASHI}

singlet state lifetime of the chromophore. The preformed excimer sites are generally negligible in dilute solutions as judged by the absence of excimer emission in a frozen matrix at $77 \mathrm{~K}$.

In solid polymers, these two conditions are not always satisfied sufficiently. The local concentration of chromophore is indeed very high whereas the restriction of segment mobility depends strongly on the nature of solid polymer. The contribution of preformed excimer sites and energy-migration processes are more important for solid polymers. Under these circumstances, it is not possible at the moment to analyse these influencing factors separately and to elucidate the causality between polymer structure and spectroscopic properties of solid polymers. Our intention is consequently, to find out the spectroscopic difference in solution and solid state phenomenologically and to discuss the difference in terms of polymer structure.

\section{EXPERIMENTAL}

\section{Materials}

Synthesis of diethyl 9-anthrylmethylmalonate (I) and 2-(9-anthrylmethyl)-1, 3-propanediol (II) have already been reported. ${ }^{7}$

\section{Bis(2-hydroxyethyl) 9-Anthrylmethylmalonate}

(III). A $3.0 \mathrm{~g}(0.09 \mathrm{~mol})$ of I and a $9 \mathrm{ml}(0.16$ mol) of ethylene glycol were heated to $100^{\circ} \mathrm{C}$ for $2 \mathrm{hr}$ in a $30 \mathrm{~m} l$ of dioxane with a small amount of sodium methoxide. After precipitating in water, recrystallization from $\mathrm{CCl}_{4}$ gave white crystals $(60 \%): \mathrm{mp} 103-104^{\circ} \mathrm{C}$; IR 3320 and $1728 \mathrm{~cm}^{-1}$; NMR $\tau=2.52(\mathrm{~s}, 2 \mathrm{H},-\mathrm{OH}), 3.58$, and $4.03(\mathrm{~m}$, $8 \mathrm{H},\left(-\mathrm{CH}_{2}-\right)_{2}, 4.24\left(\mathrm{~m}, 3 \mathrm{H},>\mathrm{CHCH}_{2}\right.$-anthryl), 7.30 $-8.30 \mathrm{ppm}(\mathrm{m}, 9 \mathrm{H}$, aromatic $\mathrm{H})$.

Bis(2-aminoethyl) 9-Anthrylmethylmalonamide (IV). Ester-amide exchange between I and ethylene diamine in methanol gave IV $(50 \%)$; $\mathrm{mp} \quad 203-206^{\circ} \mathrm{C}$ (from toluene). Polyamides derived from IV were insoluble in solvents suitable for spectroscopy.

Attempts to Prepare 2-(9-anthrylmethyl)-1,3diaminopropane $(\mathbf{V})$. Condensation of 9-formylanthracene to malonnitrile followed by catalytic hydrogenation over $\mathrm{PtO}_{2}$ gave 9-anthrylmethylmalonnitrile (40\% from 9-formylanthracene): mp 167-168 ${ }^{\circ}$; IR (CN) $2250 \mathrm{~cm}^{-1}$; NMR $\tau=$ $4.25\left(\mathrm{~m}, 3 \mathrm{H},>\mathrm{CHCH}_{2}-\right)$ and $7.30-8.30 \mathrm{ppm}$ $(\mathrm{m}, 9 \mathrm{H}$, aromatic $\mathrm{H})$. All attempts to reduce the substituted malonnitrile to $\mathbf{V}$ by catalytic hydrogenation or by metal hydrides have failed under the condition wherein an unsubstituted malonnitrile was smoothly reduced.

\section{Polymer Preparation}

Polyesters. The polycondensation of II with dithiophenol esters of dibasic acids was already reported. ${ }^{8}$

Polyurethanes. To an anisole solution of III, an anisole solution of diisocyanate was added under stirring. The molar ratio of III/diisocyanate was 1. After refluxing under stirring for $6 \mathrm{hr}$, the polymer was precipitated in methanol. Reprecipitation was repeated from anisole-methanol, then from THF-methanol. Finally, the polymer was dissolved in THF and the solution was evaporated in vacuo to give the polymer as a film.

\section{Electronic Spectroscopy}

A Hitachi EPS-III spectrometer and a Hitachi MPF-4 spectrofluorometer were used for absorption and fluorescence measurements, respectively. Fluorescence spectra were not corrected for the wavelength dependence of the sensitivity of photomultiplier and of the intensity of exciting light. For the spectroscopy of solid polymers, a thin film was cast on a quartz plate.

\section{RESULTS AND DISCUSSIONS}

\section{Polymer Syntheses}

Polycondensation of II with alkyl esters of dicarboxylic acids such as malonic, succinic, sebacic, and terephthalic acids gave low molecular weight polyesters. ${ }^{7}$ The anthryl group of II seems to bring about steric hindrance in the ester exchange process. Use of thiophenolates as activated esters improves the reactivity as briefly reported. ${ }^{8}$ Furthermore, the new process does not require any catalysts so that the contamination of resultant polymers is avoided.

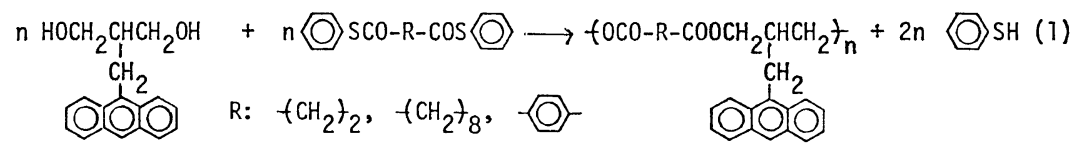


To investigate the effects of the main chain structure on the properties of polymers, the following polymers were designed.<smiles>[R]OC(=O)NCC(CC)CN=[W]</smiles>

Polyamide I

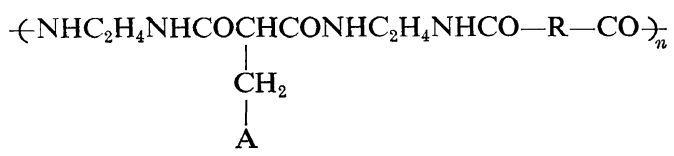

Polyamide II<smiles>[R]NC(=O)NC(=O)OCC(CC)COC(=O)[O-]</smiles>

Polyurethane I

$\left(\mathrm{OC}_{2} \mathrm{H}_{4} \mathrm{OCOCHCOOC} \mathrm{H}_{4} \mathrm{OCONH}-\mathrm{R}-\mathrm{NHCO}\right)_{n}$<smiles>CCCC</smiles>

Polyesterurethane

Among these polymers, polyamide II and polyurethane $\mathbf{I}$ are not very soluble in solvents suitable for spectroscopy and casting fllm. Although polyamide I could not be prepared, this polymer must be much more insoluble than polyamide II as judged by the stiffness of the main chain.

Polyesterurethanes were prepared as follows.<smiles>CCOC(=O)C(CC)C(C)OCC</smiles>

$$
\stackrel{\mathrm{OCN}-\mathrm{R}-\mathrm{NCO}}{\longrightarrow} \text { polyesterurethane }
$$

These polymers are soluble in THF, chloroform and dipolar aprotic solvents. The structure, abbreviation, and properties of polymers are shown in Table I. Although the molecular weights of these polymers are not very high, a transparent film can be cast from chloroform solution on a quartz plate, which can be peeled off except for $\mathrm{PE}(\mathrm{A}-\mathrm{Ph})$.

\section{Absorption Spectroscopy}

Absorption spectra of the polymers in THF solution are nearly identical with 1, 3-bisacetoxy2-(9-anthrylmethyl)propane $(\mathrm{VI})^{7}$ as a model compound. The absorption characteristics of the polymers are tabulated in Table II. Although the peaking wavelength in THF solution is almost identical for all polymers, the broadness of spectra as arbitrarily expressed by the peak/valley ratio (i.e., O.D.370nm $/$ O.D. $_{360 \mathrm{~nm}}$ ) fluctuates. As expected, the monomer model (VI) shows the sharpest absorption spectrum. Among the polymers, PE(A-2), in which the distance between anthryl

Table I. Polymer structures and properties

\begin{tabular}{|c|c|c|c|}
\hline Abbreviation & Structure $^{a}$ & $M_{w}^{\mathrm{b}}$ & $n$ \\
\hline $\mathrm{PE}(\mathrm{A}-2)$ & 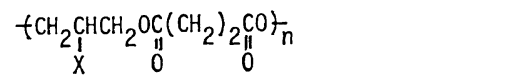 & 4,200 & $\sim 12$ \\
\hline PE(A-8) & 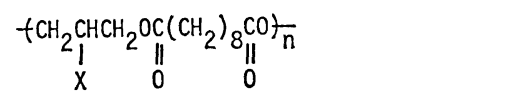 & 11,000 & $\sim 25$ \\
\hline $\mathrm{PE}(\mathrm{A}-\mathrm{Ph})$ & $\underset{x}{+\mathrm{CH}_{2} \mathrm{CHCH}_{2} \mathrm{CH}_{2} \mathrm{OC}} \underset{n}{\mathrm{n}} \mathrm{O} \underset{\mathrm{g}}{\mathrm{CO} t_{n}}$ & 1,500 & $\sim 4$ \\
\hline$P U(A-6)$ & 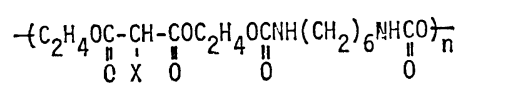 & 10,500 & $\sim 19$ \\
\hline $\mathrm{PU}(\mathrm{A}-\mathrm{Xy})$ & 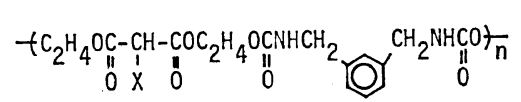 & 4,100 & $\sim 7$ \\
\hline
\end{tabular}

a X, 9-anthrylmethyl.

${ }^{\mathrm{b}}$ Estimated from the GPC elution curve (solvent, THF) calibrated for polystyrene. 
Table II. Absorption characteristics of anthryl polymers

\begin{tabular}{|c|c|c|c|c|c|}
\hline \multirow{2}{*}{$\begin{array}{c}\text { Sample } \\
\text { Monomer }\end{array}$} & \multicolumn{2}{|c|}{$\lambda_{\max }(\mathrm{nm})$} & \multirow{2}{*}{$\begin{array}{c}\varepsilon_{\max } \times 10^{-4} \\
\text { in THF }\end{array}$} & \multicolumn{2}{|c|}{ O.D. ${ }_{.370 \mathrm{~nm}}^{\mathrm{peak}} /$ O.D..$_{300 \mathrm{~nm}}^{\mathrm{val} 1 \mathrm{ey}}$} \\
\hline & THF solution & Film & & THF solution & Film \\
\hline Model (VI) & 368.0 & - & 1.04 & 3.33 & 一 \\
\hline PE (A-2) & 369.0 & 373 & 0.92 & 3.03 & 2.13 \\
\hline PE (A-8) & 369.0 & 372 & 0.97 & 3.23 & 2.56 \\
\hline $\mathrm{PE}(\mathrm{A}-\mathrm{Ph})$ & 369.3 & 374 & 0.91 & 3.03 & 2.00 \\
\hline PU (A-6) & 368.8 & 372 & 0.85 & 2.94 & 1.72 \\
\hline PU (A-Xy) & 369.0 & 373 & 0.72 & 2.94 & 2.44 \\
\hline
\end{tabular}

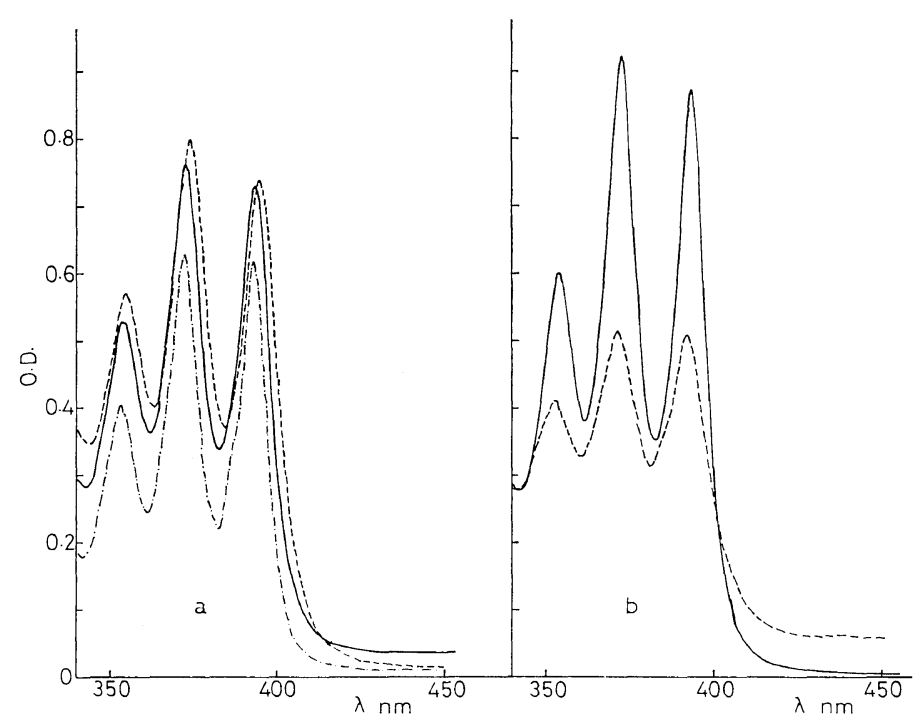

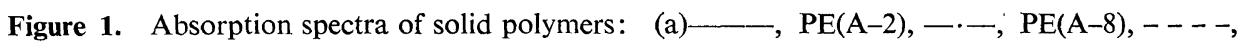
$\mathrm{PE}(\mathrm{A}-\mathrm{Ph}) ;(\mathrm{b})----, \mathrm{PU}(\mathrm{A}-6),-[\mathrm{PU}(\mathrm{A}-\mathrm{Xy})$.

groups is shorter than that in $\mathrm{PE}(\mathrm{A}-8)$, and $\mathrm{PE}$ (A-Ph), which is suspected for the interaction between anthryl and terephthalate groups, shows broader absorption spectra than that of $\mathrm{PE}(\mathrm{A}-8)$ or VI. The difference in $\varepsilon_{\max }$ seems to correspond to the sequence of the broadness. Assuming the identical absorption intensity integrated over a whole wavelength region for all samples the intensity of peaking absorption should decrease with broadening of the absorptions spectra.* The properties of the main chain seem to affect the broadness of the absorption spectrum as shown

* Since the molecular weight of polymers is not very high and the end group structure is unknown, there remain some uncertainties on $\varepsilon_{\max }$. These values were calculated for the repeating unit structure by neglecting the effects of end groups. in Figures 1 and 2. PU(A-6) shows a broad spectrum in spite of the long spacing groups between anthryl groups, indicating the ground state interactions as discussed below. With an exception of PU(A-Xy), those polymers of which absorption spectra in solution are broad give broad solid state spectra as well. The spectral difference between polymers is significant even if the difference in the base line absorption (the absorbance at the long wavelength limit) is corrected.

It is suspected that the broadening is brought about by the interactions of neighboring anthryl groups. Taking PE(A-8), which gives the sharpest spectrum as standard this standard spectrum was subtracted from the spectra of other polymers. The absorption spectra of $\mathrm{PE}(\mathrm{A}-2)$ and $\mathrm{PU}(\mathrm{A}-\mathrm{Xy})$ are rather similar to those of $\mathrm{PE}(\mathrm{A}-8)$ and spectra 


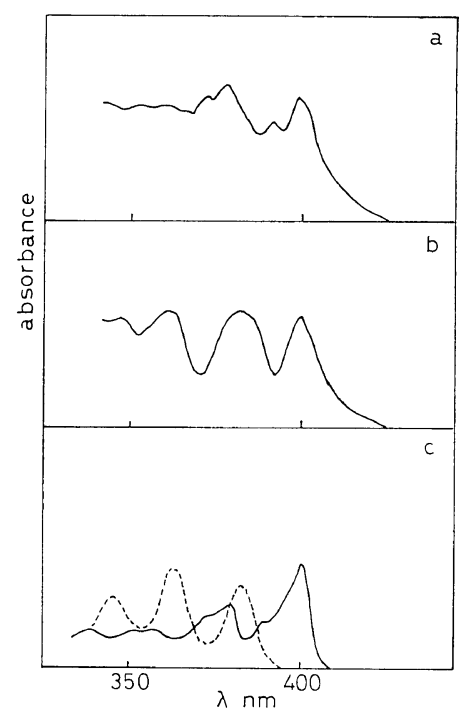

Figure 2. Difference spectra. a, b, absorption spectrum of $\mathrm{PE}(\mathrm{A}-8)$ subtracted from those of $\mathrm{PE}$ (A-Ph) (a) and PU(A-6) (b).

c, reported spectra of stable dimer (-) and sandwich dimer (-----) of anthracene.

of significant difference were not obtained. However, the difference spectra for $\mathrm{PE}(\mathrm{A}-\mathrm{Ph})$ and PU(A-6) show that the spectra of these two polymers contain the components attributable to a stable anthracene dimer or aggregates, whereas the contribution of anthracene sandwich dimer is not certain. Nevertheless, the broad absorption of PU(A-6) at about $380 \mathrm{~nm}$ and about $360 \mathrm{~nm}$ may be attributable to the sandwich dimer presented in Figure 2. The spectra of the aggregated states of anthracene have been studied in a glass matrix (isopentane-methylcyclohexane) at different temperatures. $^{9,10}$ The sandwich dimer formed by photolysing dianthracene in the glass matrix at $77 \mathrm{~K}$ is gradually converted to the stable dimer by warming up. Further warming of the matrix produces various aggregates of anthracene, depending upon temperature. The sandwich dimer of anthracene derivatives cannot be obtained directly from anthracene in solution or in glass matrices. This is observed only when the relevant anthracene photodimer is photolysed in glass matrices. The possibility of the sandwich-dimer formation in PU(A-6) therefore suggests that the polymer chain is effective in arranging two anthryl groups tightly in parallel. The main chain structure seems to play an essential role in determining the arrangement of the anthryl groups.

\section{Fluorescence Spectroscopy}

Fluorescence spectra of the polymers in dilute THF solutions show excimer emission as a broad shoulder at room temperature as has already been reported. $^{7}$ The higher molecular weights of the present samples in comparison with the previous ones result in the higher $F_{\mathrm{d}} / F_{\mathrm{m}}$ values for the present samples as shown in Table III. It has been

Table III. $F_{\mathrm{d}} / F_{\mathrm{m}}$ of various polymers at infinite dilution in THF, at room temperature

\begin{tabular}{lll}
\hline \multicolumn{1}{c}{ Polymer } & $F_{\mathrm{d}} / F_{\mathrm{m}}{ }^{\mathrm{a}}(\mathrm{DP})$ & \\
\hline PE(A-2) & $0.22(\sim 12)$ & $0.06(2-3)^{\mathrm{b}}$ \\
PE(A-8) & $0.09(\sim 25)$ & $0.04(4-5)^{\mathrm{b}}$ \\
PE(A-Ph) & $0.20(\sim 4)$ & $0.03(\sim 2)^{\mathrm{b}}$ \\
PU(A-6) & $0.10(\sim 19)$ & \\
PU(A-Xy) & $0.10(\sim 7)$ & \\
\hline
\end{tabular}

a $F_{500 \mathrm{~nm}} / F_{415 \mathrm{~nm}}$.

b Calculated from the data in ref 7 .

demonstrated that for the inter- and intramolecular exciplex formation of a polyester, the exciplex intensity increases with increasing molecular weight of polymer. $^{3}$ Although the interpolymer interactions seem to be a common feature of polymeric exciplexes, the interpolymer interactions are much weaker for polymeric excimers. In previous reports, the concentration dependence of excimer intensity in a concentration region higher than $10^{-3} M$ was reported for anthryl and naphthyl pendant polyesters ${ }^{4,7}$ whereas the exciplex intensity

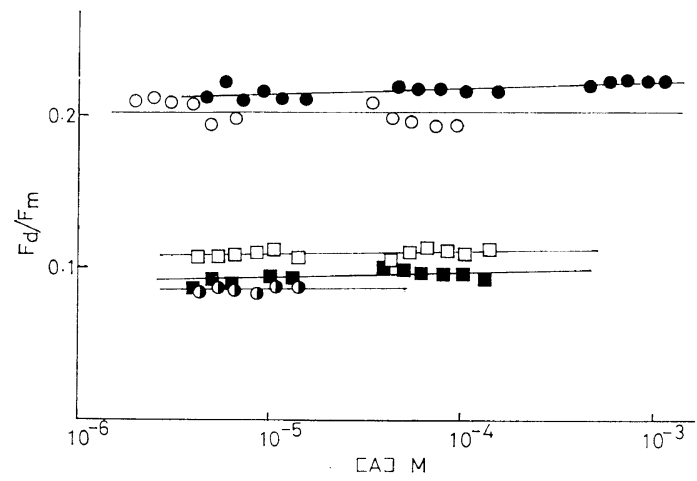

Figure 3. Concentration dependence of excimer emission in THF: $\bullet, \operatorname{PE}(\mathrm{A}-2)$;, $\mathrm{PE}(\mathrm{A}-8)$; $\mathrm{O}, \mathrm{PE}$ $(\mathrm{A}-\mathrm{Ph}) ; \square, \mathrm{PU}(\mathrm{A}-6) ; \mathbf{E}, \mathrm{PU}(\mathrm{A}-\mathrm{Xy})$. 
of polymeric systems depends on the polymer concentration in a region below $10^{-4} M .^{1,3,6}$ Detailed reinvestigation of the concentration effect confirms that the excimer intensity is constant or nearly constant below $10^{-3} M$ for the present polymers as shown in Figure 3.

The identity of the excitation spectra monitoring at $415 \mathrm{~nm}$ (the emission of monomeric anthracene) and at $500 \mathrm{~nm}$ (the excimer emission) excludes the possibility of the long-wavelength emission owing to ground state dimers or aggregates. We obtained the same results for other polymers in solution. These results are different from the excitation spectra of PE(A-1A) where $R$ in equation (1) is $>\mathrm{CHCH}_{2}$ (9-anthryl). ${ }^{7}$ The distance between anthryl groups in $\mathrm{PE}(\mathrm{A}-1 \mathrm{~A})$ is small so that the ground-state interactions of anthryl groups should not be negligible. A relatively high $F_{\mathrm{d}} / F_{\mathrm{m}}$ for $\mathrm{PE}(\mathrm{A}-\mathrm{Ph})$ might be due to the interaction between the singlet excited state of anthryl and terephthalate groups. Excimer formation between neighboring anthryl groups seems improbable for $\mathrm{PE}(\mathrm{A}-\mathrm{Ph})$ as shown by a molecular structural model. The excited-state interactions of aromatic hydrocarbons with terephthalate have been shown by fluorescence quenching experiments. ${ }^{11}$ The Stern-Volmer quenching constant of diethyl terephthalate increased with solvent polarity, indicating that the quenching reaction involved an electron transfer process from the excited aromatic hydrocarbon to terephthalate, possibly via exciplex, although the exciplex emission was not detected in the small

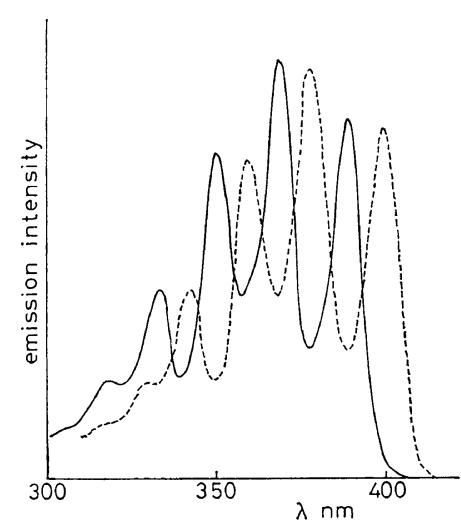

Figure 4. Excitation spectra of $\mathrm{PE}(\mathrm{A}-2)$ monitoring at $415 \mathrm{~nm}$ (- $)$ and at $500 \mathrm{~nm}(-----)$.

(The broken line spectrum is shifted towards long wavelength side by $10 \mathrm{~nm}$ to avoid overlap.) molecular system.

The solid-state fluorescence measured for polymer film cast on a quartz plate consists of broad structureless emission alone peaking at 450-460 nm except for PE(A-Ph) whose solidstate fluorescence is much weaker than others and peaks at $492 \mathrm{~nm}$. The results shown in Table IV and Figure 5 indicate that the broad emission in the solid state is in part due to the excimer but there seems to be an appreciable contribution of emission stemming from the ground-state interactions. The peak/valley ratios for the excitation spectra are smaller than those for the relevant absorption spectra in Table II, which should indicate the contribution of the ground-state aggregates. Further-

Table IV. Emission characteristic of solid state polymers

\begin{tabular}{lcc}
\hline Polymer & Emission peak $^{\mathrm{a}}$ & $\begin{array}{c}\text { Broadness of } \\
\text { excitation } \\
\text { spectrum }^{\mathrm{b}}\end{array}$ \\
\hline PE(A-2) & $\lambda_{\max }(\mathrm{nm})$ & $\boldsymbol{F}_{\sim 370} / \boldsymbol{F}_{\sim 360}$ \\
PE(A-8) & 457 & 1.73 \\
PE(A-Ph) & 454 & 1.76 \\
PU(A-6) & 492 & $\sim 1$ \\
PU(A-Xy) & 453 & 1.57 \\
\hline
\end{tabular}

a For excitation at $390 \mathrm{~nm}$, see Figure 5 b.

b For monitoring emission at peaking wavelength, see Figure 5a.

more, the relative magnitudes of the peak/valley ratios reflect qualitatively those observed for the absorption spectra presented in Table II. The abnormality of the PU(A-Xy) may be attributed to the low glass-transition temperature of the polymer $\left(1^{\circ} \mathrm{C}\right)$. The other four polymers have a $T_{\mathrm{g}}$ higher than or about equal to room temperature. $^{15}$ Details of temperature effects on spectroscopy are subjects for separate publications.

The peculiar emission from $\mathrm{PE}(\mathrm{A}-\mathrm{Ph})$ requires comment. As discussed in previous section, there are obviously interactions between the excited anthryl and terephthalate groups. However, no peaking emission of excimer/exciplex was detected in the solution system and consequently, the origin of the long wavelength emission can not be discussed. In the solid state, the peaking emission from $\mathrm{PE}(\mathrm{A}-\mathrm{Ph})$ shifts by over $30 \mathrm{~nm}$ in comparison with that of other anthryl polymers. This amount is large enough to conclude the participation of the 

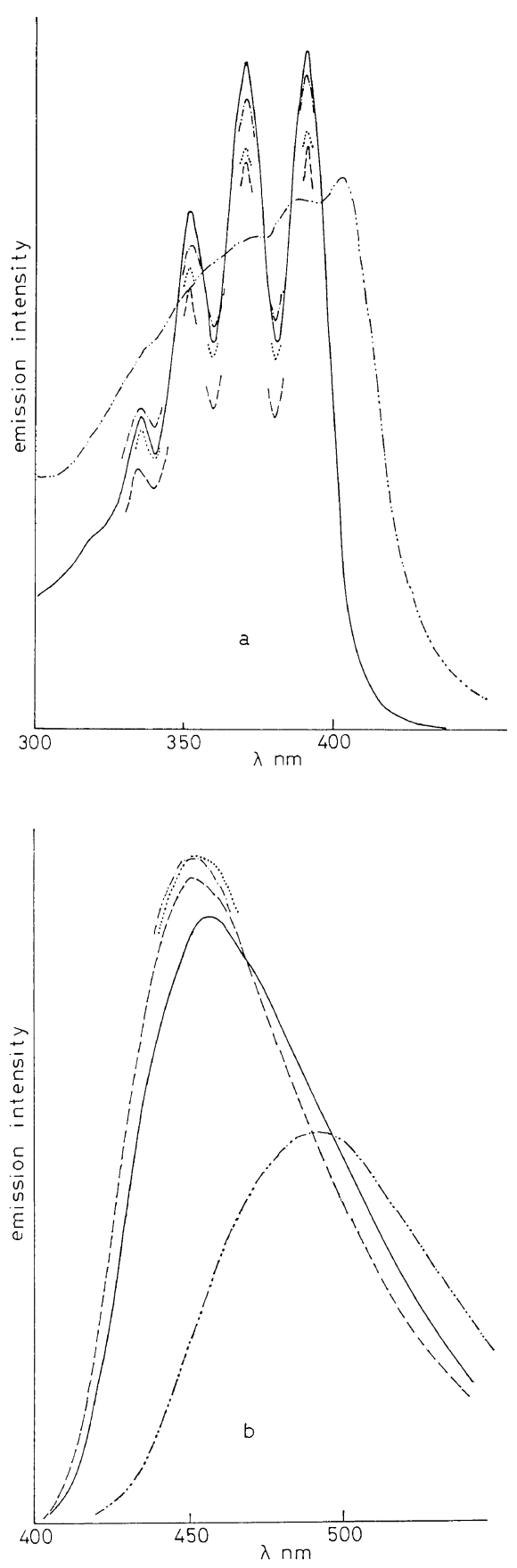

Figure 5. Fluorescence spectra of solid polymers: $\longrightarrow, \mathrm{PE}(\mathrm{A}-2)$; - - $\mathrm{PE}(\mathrm{A}-8) ;-\cdots-, \mathrm{PE}(\mathrm{A}-\mathrm{Ph})$; -.-.-, PU(A-6); …, PU(A-Xy).

(a) Excitation spectra. Monitoring at the peaking wavelength of emission.

(b) Emission spectra. Excitation at $390 \mathrm{~nm}$. terephthalate group in the excited state interactions. Both absorption and excitation spectra of $\mathrm{PE}(\mathrm{A}-\mathrm{Ph})$ suggest strong ground-state interactions. Probably, photophysical processes in $\mathrm{PE}(\mathrm{A}-\mathrm{Ph})$ will be considerably different from those in other polymers. Although the emission probability in the solid state cannot be quantitatively compared, the emission intensity of $\mathrm{PE}(\mathrm{A}-\mathrm{Ph})$ is weaker than other anthryl polymers by an order of magnitude, indicating the low emission probability of the excited complex. The strong interactions in $\mathrm{PE}(\mathrm{A}-\mathrm{Ph})$ may be related to the fact that the space formed by two anthryl groups and a terephthalate group is appropriate in size to accomodate another anthryl group. This same line of discussion was presented to explain the strong interpolymer interaction in poly (propylene terephthalate) having carbazolylbutyl side groups. ${ }^{1}$

Besides the ground-state interactions between anthryl groups, energy migration in the solid polymers can account for the entire quenching of monomer emission in all polymers. Since all measurements were carried out at room temperature, which is well below the $T_{\mathrm{g}}$ of $\mathrm{PE}(\mathrm{A}-2)$ or $\mathrm{PE}$ (A-Ph), the main-chain movement to form excimer will not be allowed at least for these two polymers. The solid-state excimer or exciplex emission may consequently result from energy migration to the preformed excimer or exciplex sites acting as energy traps or by some movements of side groups. Fluorescence depolarization measurements of the anthracene polymers in MTHF matrix at $77 \mathrm{~K}$ indicated the participation of intrapolymer energy migration $^{12}$. Energy migration must be more efficient in neat solid polymers. We are, however, not able to treat these influencing factors separately.

The excimer state of anthracene is considered as the intermediate to anthracene photodimer. This is the reason for the absence of the anthracene excimer in fluid solutions. ${ }^{14}$ A recent spectroscopic study on 9, 10-anthracenophane and $\alpha, \omega$ di(9-anthryl)alkane ${ }^{13}$ indicated that neither excimer nor monomer emission is observed when the photodimerizable conformer is preferred. For $\alpha, \omega$-di(9-anthryl)alkane, the fraction of fluorescent conformer increases and photoreaction proceeds only at elevated temperature with increasing the number of methylene groups from three to four. The results mean the importance of 


\section{S. TAZuke and N. HAYASHI}

molecular motion when the molecular conformation does not exactly match the requirement for photodimerization. In the present solid polymer systems, photodimerization proceeds only above $T_{\mathrm{g}}{ }^{15}$ whereas excimer emission is observed even below $T_{\mathrm{g}}$. Probably, the conformation leading to photodimerization is more restricted than that emitting the excimer emission. Furthermore, both head-to-head and head-to-tail encounters of 9-anthryl groups could form excimer, whereas only the later would be the precursor of photodimer.

\section{REFERENCES}

1. S. Tazuke and Y. Matsuyama, Macromolecules, $\mathbf{8}$, 280 (1975).

2. S. Tazuke, K. Sato, and F. Banba, Chem. Lett., 1321 (1975).

3. Y. Matsuyama and S. Tazuke, Polym. J., 4, 481 (1976).

4. S. Tazuke and F. Banba, Macromolecules, 9, 451 (1976).
5. S. Tazuke and Y. Matsuyama, Macromolecules, 10, 215 (1977).

6) S. Tazuke, K. Sato, and F. Banba, Macromolecules, 10, 1224 (1977).

7. S. Tazuke and F. Banba, J. Polym. Sci., Polym. Chem. Ed., 14, 2463 (1976).

8. K. Sato, N. Hayashi, and S. Tazuke, J. Polym. Sci., Polym. Lett. Ed., 15, 671 (1977)

9. E. A. Chandross and J. Ferguson, J. Chem. Phys., 45, 3554 (1966).

10. E. A. Chandross, J. Ferguson, and E. G. McRae, J. Chem. Phys., 45, 3546 (1966).

11. S. Tazuke and K. Sato, J. Phys. Chem., 80, 1727 (1976).

12. S. Tazuke, H. Tomono, N. Kitamura, K. Sato, and N. Hayashi, to be published.

13. J. Ferguson, M. Morita, and M. Puza, Chem. Phys. Lett., 49, 265 (1977).

14. J. B. Birks, "Photophysics of Aromatic Molecules." John Wiley, New York, N. Y., 1970, p 319.

15. S. Tazuke and N. Hayashi, Preprints, 26th International Congress of Pure and Applied Chemistry, Tokyo, September, 1977, p 1291. J. Poylm. Sci., Polym. Chem. Ed., in press. 